\title{
Quel(s) domaine(s) langagier(s) pour l'évaluation d'une compétence en langue? Réflexion dans le cadre du D(N)CL
}

Shaeda Isani et Micheline Hérino

\section{(2) OpenEdition}

\section{Journals}

Édition électronique

URL : http://journals.openedition.org/asp/4111

DOI : $10.4000 /$ asp. 4111

ISSN : 2108-6354

Éditeur

Groupe d'étude et de recherche en anglais de spécialité

\section{Édition imprimée}

Date de publication : 1 décembre 1994

Pagination : 249-256

ISSN : 1246-8185

\section{Référence électronique}

Shaeda Isani et Micheline Hérino, «Quel(s) domaine(s) langagier(s) pour l'évaluation d'une compétence en langue? Réflexion dans le cadre du D(N)CL », ASp [En ligne], 5-6 | 1994, mis en ligne le 20 décembre 2013, consulté le 01 mai 2019. URL : http://journals.openedition.org/asp/4111; DOI :

10.4000/asp. 4111

Ce document a été généré automatiquement le 1 mai 2019.

Tous droits réservés 


\title{
Quel(s) domaine(s) langagier(s) pour l'évaluation d'une compétence en langue? Réflexion dans le cadre du $D$ $(N) C L$
}

\author{
Shaeda Isani et Micheline Hérino
}

1 À partir du postulat qu'en matière d'évaluation il ne peut $\mathrm{y}$ avoir aucune prétention à l'exhaustivité par rapport à l'objet de l'évaluation, à savoir la langue, force est de constater que tout processus d'évaluation passe inéluctablement par une réduction arbitraire. Il convient alors de poser une problématique : par quel biais aborder l'objet de l'évaluation sans trop en trahir le caractère holistique?

2 Toute réflexion sur le(s) domaine(s) langagier(s) à cibler est tributaire des réponses apportées à deux autres questions préliminaires : quel public viser et quels objectifs d'évaluation retenir?

\section{Le public visé : un public d'adultes non spécialistes}

3 Nous n'évoquerons ici que de manière partielle la question globale des différents publics visés dans le cadre du $\mathrm{D}(\mathrm{N}) \mathrm{CL}^{1}$, et renvoyons le lecteur au numéro 3 d'ASp (Isani \& Hérino, 1993) et à la conférence du TESOL (Octobre, 1993) où cet aspect a été exposé et analysé en plus grand détail.

4 Le public principalement visé par le $\mathrm{D}(\mathrm{N}) \mathrm{CL}$ est un public d'adultes non spécialistes de langues, engagés dans la vie professionnelle, ou au stade pré-professionnel. Si le public professionnel a fait l'objet de nombreux travaux d'analyse et de réflexion, il n'en est pas de même pour le public pré-professionnel. Très brièvement nous pourrons le définir comme un public d'étudiants post-Bac dont l'intérêt pour la langue est un intérêt essentiellement utilitaire et instrumental, d'où l'utilisation du terme «non-spécialistes » 
à leur égard. Ce terme, chargé d'une connotation péjorative sera avantageusement remplacé par celui que proposent le GERAS et RANACLES, à savoir le secteur LANSAD (Langues pour spécialistes d'autres disciplines).

\section{Quels objectifs pour une évaluation en langue?}

5 À l'objectif traditionnel - la certification, pouvoir donner au candidat et à qui de droit, une idée du niveau de compétence langagière auquel se situe le candidat dans une langue donnée -nous pourrions bien entendu rajouter d'autres objectifs classiques que nous attribuons au «Proficiency Tests», aux «Achievement Tests» ou encore aux «Diagnostic Tests ». Mais il s'agit là de considérations qui sont plutôt «institutionnelles » et nous avons cherché à explorer la question sous un angle plus fondamental en nous interrogeant sur les objectifs épistémologiques à donner à l'évaluation.

\section{Compétences}

6 Si le concept d'évaluation d'une langue par le biais des connaissances ou du savoir axés sur la seule maîtrise du code linguistique perd de plus en plus d'adeptes, celui qui consiste à découper la langue en quatre savoir-faire linguistiques (tendance psychométriquestructuraliste) recueille encore l'assentiment quasi général. Il est en effet commode de pouvoir scinder la langue en deux (compréhension/expression) ou en quatre (eo/ee/co/ ce) compétences ou capacités langagières. Cependant, à cette commodité peut être faite l'objection majeure que ce découpage de la langue correspond peu à l'utilisation qui en est faite dans une situation de communication authentique. De nombreux systèmes d'évaluation existent qui recourent à l'évaluation de la langue par compétence isolée. Il s'agit de systèmes d'évaluation indirects avec tous les dangers d'approximation que cela suppose. Dans le cadre du $\mathrm{D}(\mathrm{N}) \mathrm{CL}$, la volonté de conserver à l'objet-langue son caractère holistique nous a conduit à préférer à la notion de découpage celle de compétence en langue avec tout ce que cela implique de capacité de fonctionner dans une langue d'une part, et d'ancrage de la langue dans un contexte socioculturel, d'autre part.

On a pu regretter que le terme "communication" ne figure pas dans l'intitulé du diplôme. La décision de ne pas l'y faire figurer fait partie du même processus de réflexion dans la mesure où il relève de l'évidence même que la raison d'être de toute langue réside dans le désir et la nécessité de communiquer avec autrui. Il a donc, été jugé superflu d'accoler aux termes de « compétence » et de « langue » celui de « communication. » $\mathrm{Ce}$ dernier n'en reste pas moins central à notre réflexion, dans la mesure où le $\mathrm{D}(\mathrm{N}) \mathrm{CL}$ se donne comme objectif d'évaluer la compétence d'un individu à fonctionner dans une situation de communication donnée.

\section{Quel(s) domaine(s) langagier(s) cibler?}

8 La recherche anglo-saxonne, pionnière dans ce domaine, a depuis longtemps établi les procédures d'analyse de la langue en domaines d'utilisation langagière, analyse qui a conduit à l'élaboration d'une typologie de base: English for General Purposes (EGP), English for Occupational Purposes (EOP), English for Specific Purposes (ESP). 
9 Les experts membres de l'équipe d'élaboration du $\mathrm{D}(\mathrm{N}) \mathrm{CL}$, en s'inspirant des travaux du Conseil de l'Europe et des spécifications relatives à «English for Technical Purposes» ont défini trois catégories analogues :

- Langue de communication usuelle (LCU)

- Langue de communication à usage professionnel (LCUP)

- Langue de spécialité (LS).

10 LCU et LS sont des domaines langagiers relativement faciles à définir et nous commencerons donc par là. LCU, c'est la langue de la vie quotidienne, celle qui présente la perspective la plus universaliste.

11 En revanche, il est généralement accepté que la notion de LS est propre à l'exercice d'une profession plus qu'au fait de parler une langue étrangère : nous rappellerons à cet égard que des LS peuvent parfaitement exister en langue maternelle, évidence souvent oubliée...

12 Il existe deux pôles de références par rapport aux langues de spécialité : le contexte et la fonction. Nous aimerions quelque peu nous attarder sur le contexte, en rappelant que deux approches épistémologiques se dégagent par rapport à la problématique du contexte dans les langues de spécialité: la première, que nous pourrions qualifier de tendance initiale, est très représentative des années 1970, qui ont marqué l'essor des langues de spécialité. Il s'agit d'un courant marqué par le concept de micro-contexte : on avait alors tendance à privilégier l'approche quantitative, en mettant l'accent sur l'analyse de l'occurrence et la fréquence des éléments lexicaux et morpho-syntaxiques. Cette démarche s'inscrivait dans un contexte d'objectif à court terme destiné à établir un ordre de priorité dans l'apprentissage de la langue.

13 Nous avons suivi à ce titre une expérience intéressante concernant le recrutement par le Synchrotron de Grenoble de trois techniciens de surveillance de l'accélérateur d'électrons au mois de septembre. Un des trois techniciens recrutés ayant un anglais rudimentaire, et la langue de travail au Synchrotron étant l'anglais, son embauche était conditionnée par sa capacité de surmonter, sans formation, son handicap linguistique : il devait pouvoir remplir et comprendre le "log-book» et comprendre les sessions de "workshops » hebdomadaires. Il s'agissait d'objectifs à court terme qui se limitaient essentiellement à une activité de réception et à la maîtrise du jargon du métier. Le sujet de notre observation est parvenu à ses objectifs en trois mois, estimant que le jargon essentiel se réduisait à environ 70 mots techniques, maîtrisé dès le premier mois.

14 Cependant, il est important de noter que ni l'employeur, ni le technicien n'ont prétendu à aucun moment que ces objectifs limités s'inscrivaient dans un objectif plus large visant à rendre le technicien opérationnel sur le plan de la communication. À preuve, le fait qu'une fois l'embauche confirmée, le Synchrotron a envoyé ce technicien en stage semiintensif de longue durée pour qu'il apprenne « l'anglais courant ».

15 Les exemples de ce type abondent. C'est précisément cet aspect pointu et spécifique qui a largement contribué à donner aux langues de spécialité leur image de langues ésotériques, et limitées. À ce propos, nous ne pouvons que rappeler la sonnette d'alarme tirée par Dr Peter Roe, alors English Language Officer du British Council à Paris, lors du colloque du GERAS de Montpellier en 1982 : l'auteur évoquait « the trivialisation of ESP ».

16 Effectivement, les choses ont évolué et une deuxième tendance se dessine vers les années 1980 , qui cherche à prendre quelque distance par rapport au contexte très restreint qui caractérise les langues de spécialité. Il s'agit d'une tendance qui se positionne très 
clairement dans une perspective de macro-contexte et tente de réinsérer la notion de langue de spécialité dans un contexte de communication. À ce titre nous citerons Bernard Coffey qui déclare :

There is no special language, only a principle of selection from the language to meet the purposes defined $(1984: 3)$

et Hutchinson and Waters en 1987 :

ESP is not just a matter of Science words and grammar for Scientists, Hotel words and grammar for Hotel staff and so on. [...]. There is much more to communication than just the surface features that we read and hear. $(1987: 18)$

Ces interrogations ne sont pas étrangères au GERAS : rappelons que dès 1982, le même colloque de Montpellier avait pour thème la question «Langue de spécialité ou langue pour le spécialiste?»

Dans le cadre du $\mathrm{D}(\mathrm{N}) \mathrm{CL}$, nous avons placé notre réflexion au cœur de ce débat en prenant également position contre " the trivialisation of ESP », en récusant toute notion réductrice de ce que peut constituer l'utilisation d'une langue en milieu professionnel. À cet égard il nous semble que le concept de LCUP correspond assez bien à la dynamique langagière qui caractérise « la langue de spécialisation » par rapport à « la langue de spécialité ».

Qu'entendons-nous donc par langue de communication à usage professionnel, LCUP ? Pour comprendre et pouvoir répondre, il faut analyser l'utilisation réelle qui est faite de la langue étrangère en milieu professionnel. Il existe, encore aujourd'hui, et nous pouvons en témoigner, une tendance à identifier l'utilisation d'une langue étrangère en milieu professionnel par l'aspect micro-contexte que nous venons d'évoquer, à savoir la compartimentalisation de la langue, avec focalisation pointue sur la spécificité lexicale et/ou morpho-syntaxique de telle ou telle profession ou de telle ou telle branche de profession.

La prévalence de cette vision de la langue démontre qu'il y a des idées qui ont la mort lente, car nombreuses sont les analyses de besoins langagiers réalisées sur le terrain qui démontrent que l'utilisation des langues étrangères en milieu professionnel s'inscrit dans un contexte hautement communicatif qui va bien au-delà du simple repérage de mots techniques et d'éléments morpho-syntaxiques nécessaires pour déchiffrer une notice, par exemple, de photocopieur. À ce titre, nous citerons l'ingénieur-cadre supérieur en hydraulique envoyé en formation continue accélérée en langue suite à " un gros contrat » en pays anglophone: en répondant au questionnaire destiné à recenser ses besoins langagiers, cet ingénieur a écrit : «Pour l'hydraulique, ça va très bien merci. Apprenezmoi à discuter avec mon voisin de table en anglais ", cri du cœur qui résume bien l'importance de la communication sociale et para-professionnelle dans l'utilisation d'une langue étrangère en milieu professionnel.

21 Ceci nous fournit des éléments importants pour mieux définir la LCUP. Il s'agit en effet, d'un domaine langagier hybride et fluctuant, défini par la complexité de la situation de communication en milieu professionnel : elle peut tirer vers le versant LS (discussions techniques) ou vers le versant LCU (échanges à caractère social, discussions paraprofessionnelles). Elle n'a pas, par conséquent, de statut qui lui soit propre.

\section{La notion de tâche communicative transversale}

En poussant notre analyse, nous aboutissons à la conclusion que LCUP est issue de, et ancrée dans, la LCU ; et que, contrairement à la LS, elle ne peut pas être dissociée de son 
terrain de genèse. Ceci n'est pas une constatation nouvelle, car une analyse du contenu des manuels et des méthodes d'apprentissage visant le monde professionnel le démontre clairement. Les exemples sont nombreux mais nous nous restreindrons à deux.

Le premier concerne un manuel intitulé International Business English: A course in communication skills, publié par Cambridge University Press en 1989. Dans l'introduction, les auteurs, Leo Jones et Richard Alexander, posent la question que nous nous sommes tous posée : «What is 'Business English? » et fournissent la réponse suivante :

Although there is a certain amount of vocabulary that we can describe as special

"business" vocabulary, most so-called business English is simple English used in business contexts - it is not a special language.

Notre deuxième exemple est tiré d'un manuel très utilisé par le plus grand centre de formation en langue intra-entreprise de la région grenobloise. Il s'agit d'un manuel intitulé In Business par Graham White et Margaret Khidhayar et nous attirons particulièrement l'attention sur sa date de publication, 1983, époque qui marque le changement de perspective du micro-contexte vers celle du macro-contexte. Les auteurs présentent en guise d'introduction un syllabus qui recense et classe par ordre de difficulté vingt-quatre fonctions nécessaires pour fonctionner dans le monde des affaires, fonctions que nous présentons sous forme de tableau (voir tableau 1).

Tableau 1 : Syllabus, format d'après White and Khidhayar (1983)

1. asking and answering questions/giving information/routines and habits

2. asking polite questions

3. comparing and contrasting/discussing advantages and disadvantages

4. describing things and people/measurements and calculations

5. sequencing/criticizing

6. describing trends/dealing with statistics

7. giving instructions and directions/obligation and necessity

8. describing processes

9. future plans and intentions/anticipating reactions

10. reporting and passing on information

11. personal opinions/agreeing and disagreeing/putting a point and interrupting in meetings

12. complaining/explaining and apologizing/promises and offers

13. interviewing techniques/asking for clarification/placing emphasis

14. advising and suggesting

15. comparing and contrasting/evaluating alternatives/expressing preferences 


\begin{tabular}{l} 
16. presentation techniques/dealing with objections and queries/referring to visual aids \\
17. sequencing information/evaluating information \\
\hline 18. requests and offers \\
\hline 19. certainty and uncertainty/ability and achievement \\
\hline 20. plans and intentions/rumours/probability \\
\hline 21. chairing a meeting / agreeing and disagreeing / talking about suitability \\
22. giving reasons/making recommendations \\
23. permission/prohibition/restrictions/regrets, recriminations \\
24. making arrangements/sorting out problems \\
\hline
\end{tabular}

En examinant ces fonctions nous observons que des vingt-quatre fonctions listées, une seule, le numéro 21 «chairing a meeting», peut être considérée comme n'étant pas généralement associée au domaine de la LCU - ce qui nous permet d'affirmer qu'il existe peu de tâches communicatives qui soient exclusivement du domaine de la LCUP. Cette constatation nous conduit à réitérer ce qui pourrait paraitre évident, à savoir que si la notion de "common core» existe sur le plan lexical et morpho-syntaxique, elle existe également au niveau des tâches à réaliser. Par conséquent, quelle que soit la langue de référence, LM ou LE, et quel que soit le domaine langagier, LCU ou LCUP, on peut observer et vérifier l'existence de tâches communicatives transversales.

Cette notion de tâche communicative transversale constitue un des piliers de l'évaluation de la compétence en langue, une langue qui correspond à la dynamique de « la langue en action ", telle qu'elle est utilisée sur le terrain. Ainsi, le DNCL portera sur la capacité d'un candidat à pouvoir réaliser les mêmes opérations et accomplir les mêmes tâches en LE qu'il réalise normalement en LM, en LCU qu'en LCUP. Comme tâche complexe nous avons choisi de cibler celle du traitement de l'information, activité débordante qui sous-tend la plupart des tâches communicatives. Le traitement de l'information se décomposant en trois activités : la réception, le traitement et la restitution.

L'objectif de l'évaluation sera donc de mesurer la capacité du candidat à accomplir la tâche communicative complexe en mettant en œuvre les trois opérations et leurs différentes composantes de la compétence de communication.

Ayant, nous l'espérons, pu démontrer l'étroite imbrication et le recouvrement LCU/LCUP (voir Isani \& Hérino 1994), nous allons maintenant tenter de répondre à la question que nous nous entendons souvent poser: la différence LS et LCU nous paraît claire, mais existe-t-il une différence entre LCU et LCUP ? La réponse est oui. Malgré les nombreuses zones de recouvrement qui existent pour rapprocher la LCU de la LCUP, il y a une importante différence qui les distingue, celle de la situation de communication.

Dell Hymes en 1982 fut parmi les premiers à définir la compétence en langue comme étant inféodée à la situation de communication et ses différents paramètres définitoires, le lieu, l'interlocuteur, le canal, l'objet de l'échange... Toute modification touchant à un des paramètres engendre des variations sur le code socioculturel qui conditionne la 
nouvelle situation de communication, ce qui à son tour conditionne le type de discours et le comportement langagier considérés appropriés ou inappropriés par rapport à cette situation.

Nous nous rapprochons ici de la notion de "speech community ", à l'intérieur de laquelle il existe un certain nombre de conventions qui régit non seulement ce qui est considéré comme discours approprié et ce qui ne l'est pas, mais comprend également le code socioculturel relatif au statut social, « la position que tient une personne dans la structure sociale d'une communauté » et son rôle social, «les modes de comportement que la communauté attend d'une personne d'un certain statut » (Crystal 1987 : 40).

Dans le contexte des statut et rôle sociaux, nous mentionnerons également les travaux d'Erving Goffman dont les titres mêmes sont très évocateurs par rapport à nos propos. En effet, il parle « des rites de l'interaction ", de " la mise en scène de la vie quotidienne », de «la présentation du soi » et "des relations en public». Ses théories sur la notion de représentation et de la face engendrent celle de la façade sociale qui à son tour est liée à celle du décor. Il est aisé de comprendre l'importance du rôle que peut jouer le langage dans ce contexte hautement socioculturel.

l est important de noter qu'un interlocuteur n'est jamais figé dans un statut et rôle social, qu'il n'adhère pas à une seule communauté discursive et qu'il ne se trouve pas dans un seul type de situation de communication. Bien au contraire, les modifications qui s'opèrent sans cesse au niveau de la situation de communication, du rôle et du statut de l'interlocuteur, engendrent à chaque fois, obéissance à des nouvelles conventions régies par un nouveau code socioculturel.

Or, une des distinctions majeures entre LCU et LCUP réside dans la situation de communication et, si d'autres composantes de la situation de communication peuvent rester quasiment les mêmes (le lieu et l'interlocuteur, par exemple), il n'est pas de même en ce qui concerne l'objet de la communication. Deux principes tirés du domaine juridique peuvent nous aider à mieux saisir ce qui distingue l'objet de communication en LCU par rapport à celui de LCUP : il s'agit du principe de l'obligation de moyens et celui de l'obligation de résultat. Dans le premier cas, il incombe au professionnel seulement de mettre en œuvre tous les moyens à sa disposition pour aboutir à un résultat, alors que dans le deuxième cas il est tenu d'obtenir le résultat.

Sans vouloir établir des généralisations inutiles, on peut néanmoins dire que la situation de communication non professionnelle est très souvent moins « tendue » et subit moins de pressions parce qu'elle est davantage marquée par le principe de la simple obligation de moyens. Par contre, en LCUP, l'objet de communication est étroitement lié à une forte notion d'enjeu qui est générée par le fait que se dessine toujours en toile de fond le principe de l'obligation de résultat si caractéristique du monde professionnel. Ce principe d'obligation de résultat pèse sur les échanges en milieu professionnel et modifie considérablement la nature de la situation de communication qui est désormais transformée en situation de communication à risque, redéfinissant ainsi les conventions qui gouvernent les rôle et statut sociaux de l'interlocuteur et le code socioculturel qui les régit.

Nous aimerions illustrer ces propos par un exemple concret, celui d'un cadre d'entreprise qui possède un bagage langagier limité en langue étrangère $\mathrm{X}$. Or, selon la situation de communication dans laquelle il s'aventure à exploiter ses maigres réserves langagières, et en fonction des statuts et rôles sociaux qu'il présente, il sera bien ou mal perçu. 

notre cadre d'entreprise peut se voir encouragé, et même félicité, pour l'utilisation qu'il fait du peu de bagage qu'il possède de la langue étrangère (cf. le principe de l'obligation de moyens). En effet, dans une situation de communication sans enjeu professionnel, les échanges peuvent avoir lieu dans un climat marqué par une relative absence de « pression » et donc de « tension».

Il n'en est pas ainsi dans une situation de communication professionnelle marquée par le principe de l'obligation de résultat. Si nous prenons le cas du même interlocuteur, s'exprimant avec le même bagage langagier limité, mais revêtant les statut et rôle sociaux d'un cadre d'entreprise voyageant pour le compte de sa société, en situation de communication définie par la LCUP par conséquent, nous observons qu'il est perçu comme un interlocuteur « défaillant » dont les capacités langagières limitées choquent, voire irritent parce que, premièrement elles s'inscrivent en faux par rapport à ce que l'on pourrait attendre de par sa "façade sociale» en situation de communication professionnelle, et deuxièmement, en raison de la nature complexe du phénomène interactionnel, elles sont perçues par l'interlocuteur professionnel comme pouvant compromettre sa propre obligation de résultat.

Ceci nous conduit donc à la conclusion, que dans une situation de communication dominée par LCU, il y plus d'indulgence, voire de laxisme, par rapport à une maîtrise limitée de la langue et le seuil d'acceptabilité tolère un degré d'approximation élevé. En revanche, les représentations sont bien plus exigeantes en situation de communication professionnelle dominée par LCUP, où la notion d'enjeu et de risque place la barre du seuil d'acceptabilité bien plus haut.

Nous espérons avoir pu apporter quelques réponses aux questions qui subsistent concernant les trois domaines langagiers, la LCU, la LCUP et la LS. Notre mot de conclusion sera donc, que les éléments importants qui les rapprochent, ainsi que ceux qui les différencient, ne se situent pas forcement au premier degré d'observabilité mais sont à découvrir bien au-delà des mots, au niveau de la compétence de communication en langue et $\mathrm{du}$ comportement langagier, ce qui explique notre décision de centrer l'évaluation de la compétence en langue dans le cadre du DNCL sur le domaine langagier défini par la LCUP.

\section{BIBLIOGRAPHIE}

Coffey, B. 1984. « ESP: English for Specific Purposes ». Language Teaching 17/1.

Crystal, D. 1987. The Cambridge Encyclopedia of Language. Cambridge : Cambridge University Press.

Goffman, E. 1973. La mise en scène de la vie quotidienne, vols I \& II. Traduit de l'anglais par Alain Kihm. Paris : Les Éditions de Minuit.

Goffman, E. 1974. Les rites de l'interaction. Traduit de l'anglais par Alain Kihm. Paris : Les Éditions de Minuit. 
Hutchinson, T. et A. Waters. 1987. English for Specific Purposes: A Learning-Centred Approach. Cambridge : Cambridge University Press.

Isani, S. et M. Hérino. 1994. « La notion de scénario comme outil d'évaluation ». ASp 3, 101-121.

Jones, L. et R. Alexander. 1989. International Business English: A Course in communication skills. Cambridge : Cambridge University Press.

Roe, P. 1981. « Dimensions of difficulty in ESP ». Actes du IV Colloque du GERAS, Bordeaux, 17-24.

White, G. et M. Khidhayar. 1983. In Business. Londres : Harrap.

\section{NOTES}

1. Initialement nommé Diplôme national de compétence en langue, le $\mathrm{D}(\mathrm{N}) \mathrm{CL}$, dans le décret qui l'institue sous la double tutelle des Ministères de l'Éducation nationale et de l'Enseignement supérieur, s'appellera Diplôme de compétence en langue.

\section{RÉSUMÉS}

Cet article explique la problématique du choix de domaine langagier dans l'évaluation et répond à deux questions souvent posées, d'une part l'absence de la composante langue de spécialité dans le $\mathrm{D}(\mathrm{N}) \mathrm{CL}$ et, d'autre part, la différence entre la langue de communication usuelle et la langue de communication à usage professionnel.

This article explains the problem of selecting a particular field of language use for evaluation purposes and answers two frequently asked questions: first, why was ESP not included as one of the components of the DNCL and, secondly, what difference is there between English for General Purposes (EGP) and English for Occupational Purposes (EOP)?

\section{INDEX}

Mots-clés : communication, langue de communication usuelle, langue de communication à usage professionnel, langue de spécialité, tâche de communication

Keywords : common core task, communicative situation, EGP, EOP, English for Specific Purposes

\section{AUTEURS}

\section{SHAEDA ISANI}

Shaeda Isani enseigne à l'Université Stendhal Grenoble 3. shaeda.isani@u-grenoble3.fr

\section{MICHELINE HÉRINO}

Micheline Hérino enseigne à l'Université Stendhal Grenoble 3. 